\title{
Preliminary thoughts on research in medical humanities
}

\author{
Xiaojing Yun*, Jiawei Guo, Haihong Qian* \\ School of Basic Medical Sciences, Fudan University, Shanghai, China.
}

\begin{abstract}
Summary Medical humanities (MH) is an interdisciplinary field of medicine which includes the humanities (literature, philosophy, ethics, history, and religion), social sciences (anthropology, cultural studies, psychology, sociology, and health geography), and the arts (literature, theater, film, and visual arts) and their application to medical education and practice. Studies of MH should not be limited to theoretical discussions. Research results must be translated into use of methodologies to formulate medical policies, guide clinical practices, and help resolve physical or mental problems. MH has a critical role in addressing medicine-related issues, such as human cloning legislation and the treatment of Ebola virus infection. Recently, MH has also been included in the "Healthy China 2030" project, indicating that MH has garnered more attention in China. Medical colleges, research institutes, and non-profit organizations are focusing on MH studies. Over the past few years, financial support for MH studies has also increased. Although the development of MH currently lags behind medicine and health sciences, MH has promise.
\end{abstract}

Keywords: Medical humanities, medical education, medical humanities research, China

\section{Introduction}

Medical humanities $(\mathrm{MH})$ provides a humanistic problem-based approach to medicine aiming at influencing its nature and practice (1). A combination of medicine and the humanities, $\mathrm{MH}$ has arisen along with the rapid development of medicine and health sciences since the 1960s (2). With MH increasingly characterized as an interdisciplinary endeavor that draws on the creative and intellectual strengths of diverse disciplines in pursuit of improved medical education and practice, $\mathrm{MH}$ plays an important role. However, the worldwide development of $\mathrm{MH}$ is still relatively slow. In China, $\mathrm{MH}$ started in $80 \mathrm{~s}$ and has yielded some certain results thus far (3). However, $\mathrm{MH}$ in China is still mainly focused on $\mathrm{MH}$ education and courses. Although studies of $\mathrm{MH}$ have been conducted, most are theoretical discussions, limiting their ability to

Released online in J-STAGE as advance publication April 29, 2017.

*Address correspondence to:

Drs. Xiaojing Yun and Haihong Qian, School of Basic Medical Sciences, Fudan University, 275\# No.131, Dong'an Rd, Xujiahui District, Shanghai 200032, China.

E-mail: xjyun@fudan.edu.cn (Yun XJ); hhqian@fudan.edu.cn (Qian $\mathrm{HH})$ guide health policy or clinical practice. Like medicine, $\mathrm{MH}$ must be translated into use of methodologies to formulate medical policies, guide clinical practices, and help resolve physical or mental problems.

\section{The use of MH in medical policy-making}

Cloning was the most controversial medical topic at the beginning of this century (4). With the development of cloning technology, human cloning could be performed. As promising as it may be scientifically, human cloning poses a variety of problems that cannot be resolved through an approach defined by narrow disciplinary boundaries but rather through an approach grounded in multiple fields like MH. An anthropological concern is that human cloning violates the principle of sexual reproduction and instead represents asexual reproduction, provoking philosophical and religious debates. In addition, human cloning would disrupt the normal ethics of families. In existing human reproduction, the reproductive model depends on male and female contributions, but cloning only requires genetic material from a single parent, which means that humans can continue to reproduce as long as there are women (5). Most countries have prohibited human reproductive cloning experiments through legislation or statements since around 2001 (6). MH studies on 
cloning limited the irrational use of the technology and avoided its unintended consequences.

MH also guides government policies. In 2016, the Chinese Government launched the "Healthy China 2030" project, a bold declaration that made public health a precondition for all future economic and social development (7). An "expanded concept of health" has also been put forth with an emphasis on medical science as well as on issues of public health, nutrition, and tobacco cessation. A recent effort of this project was the banning of cigarette smoking in public areas. Smoking is not limited to medicine since it also involves sociology and public communication \& education. As of March 1st, smoking was banned in public spaces indoors, workplaces, mass transport, and other areas of Shanghai, following Beijing and Shenzhen (8). In support of Shanghai's effort, Bernhard Schwartländer, the World Health Organization's (WHO) Representative in China, remarked that "Making Shanghai smokefree is a necessary decision and China's responsibility as great power." Widespread public support, extensive public education, and public participation are key to the effective prohibition of smoking (9), according to Professor Hua Fu, Director of the Institute of Health Communication, Fudan University. The Institute, sponsored by Fudan University's School of Public Health in line with the concept of resource integration and multidisciplinary cooperation, has conducted innovative research on major health problems in the course of social and economic development. In March 2016, the Institute set up a tobacco control research center on the basis of years of tobacco control work and it launched the smoking-free Shanghai Health Communication Project under the leadership of the city government, which in turn lead to the final ban on smoking (10).

\section{The use of MH to guide clinical practices}

$\mathrm{MH}$ also plays a critical part in clinical practices. One example of this is the response to the Ebola epidemic in West Africa. There was no cure for the disease until mid-2014 when ZMapp, an experimental drug to combat the Ebola virus, was used on a limited basis. In 2014, ZMapp was used to treat 7 individuals infected with the Ebola virus, and 2 of those individuals died (11). Due to the small sample size, there is no evidence that the drug is significantly efficacious. ZMapp has demonstrated some benefit thus far, but the drug does not meet the pre-specified statistical threshold for efficacy or further studies (12).

In accordance with those findings, some experts have contended that any use of drugs that have yet to be tested for safety in large-scale human trials is unethical and potentially catastrophic. Despite widespread disapproval of ZMapp, Peter Piot, co-discoverer of the Ebola virus, supported its use as an experimental drug, given the unprecedented epidemic in West Africa. From an $\mathrm{MH}$ perspective, the unprecedented use of ZMapp is grounded in the principle of compassion, i.e. when a patient is faced with a life-threatening condition, the patient can receive an untested intervention in emergency circumstances (13). Therefore, the WHO convened a panel on August 11, 2014 to discuss and assess the ethical implications of use of unregistered interventions that have shown promising results in the laboratory and in animal models but that have not yet been evaluated for safety and efficacy in humans. The panel members unanimously concluded that on both ethical and evidentiary grounds the use of unregistered interventions, e.g. ZMapp, would be acceptable, provided that certain conditions are met. In reaching this conclusion, the panel members were mindful that their decision was a departure from the wellestablished, historically evolved system of regulation and governance of therapies and interventions, an unprecedented event in the history of $\mathrm{MH}$ (14).

Just as Dr. E.L Trudeau's epitaph famously declares, "To cure sometimes, to relieve often, to comfort always" (15). In clinical practices, humane care is considered to be equally as important as the treatment itself. MH education has received a great deal of attention in clinical training of doctors. $\mathrm{MH}$ heightens awareness of and appreciation for the "patient as a whole" in medicine. Medical students are also required to graduate with a greater understanding of the art of medicine, the importance of the doctor-patient relationship, the spiritual and emotional dimensions of disease, and the human experience of illness. Exploring the many meanings of the word 'critical', Viney et al. argued for a critical MH characterized by: $(i)$ a widening of the aspects of 'the medical' beyond the primal scene of the clinical encounter; ( $i i)$ greater focus not simply on the context and experience of healthcare but also on their composition at a variety of levels; (iii) closer engagement with studies of critical theory and disability, activist politics, and other relevant areas; (iv) a belief that the arts, humanities, and social sciences are best regarded not as in service or in opposition to the clinical and life sciences but are productively entangled in a 'biomedical culture'; and, following on from this, (v) a commitment to new types of interdisciplinary and intersectional collaboration (16).

\section{Support for MH}

At present, medical schools or institutes, such as the Yale School of Medicine and the NYU School of Medicine, mainly provide $\mathrm{MH}$ education or conduct $\mathrm{MH}$ research. This requires that $\mathrm{MH}$ programs consist of experts with different backgrounds. For example, the Medical Humanities Program at Baylor University consists of experts from different backgrounds, such as clinical medicine, philosophy, and art. Think tanks 
Table 1. Top 20 Institutions ranking in the top 1\% on Essential Science Indicators (ESI) in clinical medicine and their ranks in social sciences, general in China

\begin{tabular}{|c|c|c|c|}
\hline No. & University & Clinical Medicine & Social Sciences, General \\
\hline 1 & Shanghai Jiaotong University & 184 & 525 \\
\hline 2 & Sun Yat-sen University & 253 & 661 \\
\hline 3 & Peking Union Medical College & 268 & 833 \\
\hline 4 & Fudan University & 270 & 416 \\
\hline 5 & Peking University & 280 & 258 \\
\hline 6 & Zhejiang University & 405 & 622 \\
\hline 7 & Capital Medical University & 411 & - \\
\hline 8 & Sichuan University & 439 & 1130 \\
\hline 9 & Nanjing Medical University & 469 & - \\
\hline 10 & Huazhong University of Science and Technology & 477 & 938 \\
\hline 11 & The Second Military Medical University & 479 & - \\
\hline 12 & Shandong University & 509 & 1008 \\
\hline 13 & The Fourth Military Medical University & 522 & - \\
\hline 14 & Central South University & 533 & 1170 \\
\hline 15 & China Medical University & 607 & - \\
\hline 16 & Nanjing University & 639 & 894 \\
\hline 17 & Southern Medical University & 675 & - \\
\hline 18 & The Third Military Medical University & 677 & - \\
\hline 19 & Harbin Medical University & 682 & - \\
\hline 20 & Wuhan University & 709 & 802 \\
\hline
\end{tabular}

can also fulfill this role (17). In a general sense, the most important function of a think tank is to provide the government with intellectual support to assist with policy-making. However, think tanks with an $\mathrm{MH}$ background serve in additional roles. In addition to providing theoretical support, think tanks involved in $\mathrm{MH}$ help to address specific aspects of medical and health problems and to educate the public about the concept of $\mathrm{MH}$. In other words, these think tanks should become a database for government decision-making as well as a platform for the spread of $\mathrm{MH}$ in medical and health practices.

In around the year 2000, medical colleges began to increasingly join with full-fledged universities in China. For example, Shanghai Medical College was incorporated into Fudan University, and Beijing Medical University was incorporated into Peking University. This trend has greatly benefited the development of $\mathrm{MH}$ because of academic exchanges between medical and humanities faculties at universities. Over the past few years, more and more centers and institutes of $\mathrm{MH}$ have been launched. The Institute of Medical Humanities was established at Peking University in 2008, and it mainly focuses on studying the value of life through an interdisciplinary approach. An online course entitled Introduction to Medical Humanities was created at Fudan University in 2015, and it has attracted considerable attention from around the country in the two years that followed. This course is taught by academics, medical scientists, philosophers, educators, and doctors. A data analysis has indicated that almost half a million students from 140 universities have benefited from this course, reflecting the wide demand for knowledge on $\mathrm{MH}$. Universities undoubtedly provide better support to the humanities. The current authors examined the top 20 Chinese educational institutions ranking in the top $1 \%$ on Essential Science Indicators (ESI) in clinical medicine. Thirteen of the institutions were universities and 7 were medical colleges. All 13 of the universities ranked in the top $1 \%$ on ESI in social sciences (general) while only one medical college ranked in the top $1 \%$ in this field (Table 1). This does not preclude $\mathrm{MH}$ from being improved at medical colleges. In fact, $\mathrm{MH}$ education needs to be provided and $\mathrm{MH}$ research needs to be conducted both in medical colleges and in universities with a medical college. The equivalent academic strength of medical and social sciences offers additional options and chances to develop $\mathrm{MH}$.

$\mathrm{MH}$ is not limited to universities and colleges. Some non-governmental organizations participate in policy-making and conduct research. In China, a nonprofit organization entitled the China-Dolls Center for Rare Disorders (CCRD, formerly known as the ChinaDolls Care and Support Association) was founded by patients with osteogenesis imperfecta (OI) and other rare disorders in 2008. The CCRD provides medical rehabilitation and individualized support to people with OI and other rare disorders, it facilitates selfexploration and empowerment, it engages in public advocacy and policy-making, and it conducts research. The CCRD is working to eliminate the stigma of having a rare disorder, to improve social security, to sponsor legislation, and to promote equal access to medication, education, employment, and society for patients (18).

The development of $\mathrm{MH}$ is lagging behind medicine, as was mentioned earlier, and the same is true for financial support. Financial support for $\mathrm{MH}$ comes mainly from government investment or private donations. More attention has been paid to $\mathrm{MH}$ 
research in recent years. In 2011, the Royal Society of Edinburgh and the Scottish Government funded the launch of the Medical Humanities Research Network Scotland (MHRNS) in order to enable greater and more sustained collaborative research on $\mathrm{MH}$ in Scotland (19). The NIH also devised special support for MH. The Glasgow University Medical Humanities Network, supported by the Wellcome Trust, acts as a forum to connect individuals working across a range of disciplines and practices at the University of Glasgow, providing an intersection of medicine, culture, and the arts and humanities. As a global charitable foundation, the Wellcome Trust supports areas such as biomedical science, population health, the humanities, and social sciences. Private endowments are also facilitating $\mathrm{MH}$ research at institutions such as the University of California, San Francisco (20). The National Science Foundation of China or the National Natural Science Fund of China need to launch special projects to support research on $\mathrm{MH}$ in China.

\section{Conclusion}

Critical MH is an approach that argues that the arts and humanities have more to offer to healthcare than simply improving medical education. It proposes that the arts and humanities offer different ways of thinking about human history, culture, behavior and experience that can be used to dissect, critique, and influence healthcare practices and priorities. $\mathrm{MH}$ balances medical technology and humanity. On one hand, MH limits the irrational use of technology and it avoids unintended consequences of that use. On the other hand, MH helps to formulate medical policies, guide clinical practices, and resolve physical or mental problems. Achieving such an ambitious goal requires the establishment of an interdisciplinary field in which different people can actually interact and produce worthwhile results. Institutions and organizations are increasingly interested in $\mathrm{MH}$ education and research, and financial support is increasingly being given to $\mathrm{MH}$. These trends are promising. Universities should promote $\mathrm{MH}$ because they have sufficient resources to do so in terms of medical, social science, and humanities faculties. More financial funds should be allocated to $\mathrm{MH}$. Much is required of $\mathrm{MH}$, and much is expected of it. There is still much work to do.

\section{References}

1. Chiapperino L, Boniolo G. Rethinking medical humanities. J Med Humanit. 2014; 35:377-387.

2. Fox D. Who we are: The political origins of the medical humanities. Theor Med Bioeth. 1985; 6:327-342.
3. Zhang D. Dilemma and challenge: A review on the medical humanities in China. Medicine \& Philosophy. 2001; 22:10-13. (in Chinese)

4. Nippert I. The pros and cons of human therapeutic cloning in the public debate. J Biotechnol. 2002; 98:53-60.

5. McGee G. The Perfect Baby: Parenthood in the New World of Cloning and Genetics. Rowan \& Littlefield. Washington DC, USA, 2001.

6. The Center for Bioethics \& Human Dignity. Why human cloning must be banned now. https://cbhd.org/content/ why-human-cloning-must-be-banned-now (accessed April 6, 2002).

7. ChinaDaily. Draft law aims to prevent diseases. http://usa. chinadaily.com.cn/epaper/2017-03/07/content_28462064. htm (accessed March 7, 2017)

8. ChinaNews. www.chinanews.com/sh/2017/0214/8149456.shtml (accessed February 14, 2017). (in Chinese)

9. Xinhua Net. http://sh.xinhuanet.com/2017-02/15/ c_136058305.htm (accessed Feb 15, 2017). (in Chinese)

10. Fudan News. http://news.fudan.edu.cn/2016/0412/41220. html (accessed April 12, 2016). (in Chinese)

11. McCarthy M. US signs contract with ZMapp maker to accelerate development of the Ebola drug. BMJ. 2014; 349:g5488.

12. PREVAIL II Writing Group, Multi-National PREVAIL II Study Team, Davey RT Jr, Dodd L, Proschan MA, Neaton J, Neuhaus Nordwall J, Koopmeiners JS, Beigel J, Tierney J, Lane HC, Fauci AS, Massaquoi MBF, Sahr F, Malvy D. A randomized, controlled trial of ZMapp for Ebola virus infection. N Engl J Med. 2016; 375:1448-1456.

13. Landry JT, Foreman T, Kekewich M. Reconsidering the ethical permissibility of the use of unregistered interventions against Ebola virus disease. Camb Q Healthc Ethics. 2015; 24:366-369.

14. WHO. Ethical considerations for use of unregistered interventions for Ebola viral disease: Report of an advisory panel to WHO. http://www.who.int/csr/resources/ publications/ebola/ethical-considerations/en/ (accessed Feb 15, 2017)

15. Gordon J. Medical humanities: To cure sometimes, to relieve often, to comfort always. Med J Australia. 2005; $182: 5-8$

16. Murray J. Development of a medical humanities program at Dalhousie University Faculty of Medicine, Nova Scotia, Canada, 1992-2003. Acad Med. 2003; 78:1020-1023.

17. Kong XJ, Zhao MJ, Liu WW, Wen DG. Establishing a new type of university think tanks: A new way of medical humanities studies. Educ Sci. 2015; 31:47-53. (in Chinese)

18. China-Dolls Center for Rare Disorders. www.chinadolls. org.cn. (accessed Feb 15, 2017) (in Chinese)

19. Medical Humanities Research Network Scotland (MHRNS). http://www.gla.ac.uk/schools/critical/research/ fundedresearchprojects/mhrns/ (accessed Feb 15, 2017)

20. Center for Humanities and Health Sciences. http://www. medicalhumanities.ucsf.edu/Center_Hum_HealthSci.html (accessed Feb 15, 2017)

(Received April 5, 2017; Revised April 17, 2017; Accepted April 18, 2017) 